\title{
Multiscale Topological Trajectory Classification with Persistent Homology
}

\author{
Florian T. Pokorny \\ Centre for Autonomous Systems, CSC \\ KTH Royal Institute of Technology, Sweden
}

\author{
Majd Hawasly \\ IPAB, School of Informatics \\ University of Edinburgh, UK
}

\author{
Subramanian Ramamoorthy \\ IPAB, School of Informatics \\ University of Edinburgh, UK
}

\begin{abstract}
Topological approaches to studying equivalence classes of trajectories in a configuration space have recently received attention in robotics since they allow a robot to reason about trajectories at a high level of abstraction. While recent work has approached the problem of topological motion planning under the assumption that the configuration space and obstacles within it are explicitly described in a noise-free manner, we focus on trajectory classification and present a samplingbased approach which can handle noise, which is applicable to general configuration spaces and which relies only on the availability of collision free samples. Unlike previous samplingbased approaches in robotics which use graphs to capture information about the path-connectedness of a configuration space, we construct a multiscale approximation of neighborhoods of the collision free configurations based on filtrations of simplicial complexes. Our approach thereby extracts additional homological information which is essential for a topological trajectory classification. By computing a basis for the first persistent homology groups, we obtain a multiscale classification algorithm for trajectories in configuration spaces of arbitrary dimension. We furthermore show how an augmented filtration of simplicial complexes based on a cost function can be defined to incorporate additional constraints. We present an evaluation of our approach in 2, 3, 4 and 6 dimensional configuration spaces in simulation and using a Baxter robot.
\end{abstract}

\section{INTRODUCTION}

The problem of determining a continuous path $\gamma:[0,1] \rightarrow$ $\mathcal{C}_{f}$ between two points in the collision free subset $\mathcal{C}_{f}$ of some configuration space $\mathcal{C} \subseteq \mathbb{R}^{d}$ is an important classical motion planning problem. Since, in realistic robotics applications, an explicit description of $\mathcal{C}_{f}$ is often not available, popular algorithms such as Rapidly-exploring Random Trees (RRT) and Probabilistic Roadmaps (PRM) [25, 24, 20] are based on the idea of utilizing a set of random samples $X \subset \mathcal{C}_{f}$ to construct a graph $\Gamma$ with vertices in $\mathcal{C}_{f}$ and where edges correspond to local paths which can be determined by a local path planner. The graph $\Gamma$ can then be used to efficiently carry out motion planning. If $\mathcal{C}_{f}$ is a tame space, $\Gamma$, for sufficiently large $X$, provides an approximation of $\mathcal{C}_{f}$ which allows us to answer basic questions about the path-connectivity of $\mathcal{C}_{f}$. However, $\Gamma$ does typically not capture higher order homological information.

In this paper, we propose a novel approach based on filtrations $\mathcal{F}=\left\{\mathcal{F}_{r}: r \geqslant 0\right\}$ of simplicial complexes defined in terms of random samples $X \subset \mathcal{C}_{f}$. From such filtrations, we then extract higher-order topological information for the purpose of understanding and classifying equivalence classes of trajectories in $\mathcal{C}_{f}$. Given a sufficiently good approximation of $\mathcal{C}_{f}$ by $\mathcal{F}_{r}$, our approach yields a finite set of equivalence classes with the property that no trajectory belonging to one equivalence class can be continuously deformed to any trajectory in any of the other equivalence classes. Our filtration $\mathcal{F}$ is based on Delaunay-Čech complexes which depend on a scale parameter $r$ and which have very recently been proven [3] to provide a homotopy-equivalent reconstruction of the space $X_{r}=\bigcup_{x \in X} \mathbb{B}_{r}(x)$ [3], where $\mathbb{B}_{r}(x)=\left\{y \in \mathbb{R}^{d}: \| x-\right.$ $y \| \leqslant r\}$. Our work utilizes persistent homology $[15,11,16]$ which generalizes classical homology groups to a multiscale setting - meaning that we are able to compute topological information about the analogue $\mathcal{F}_{r}$ of $\Gamma$ for all scales $r \geqslant 0$ simultaneously without having to choose a particular scale upfront. Additionally, the 1-skeleton $\mathcal{F}_{r}^{1} \subseteq \mathcal{F}_{r}$ is a graph which can be used for path-planning. When a cost function $c: \mathcal{C}_{f} \rightarrow \mathbb{R}$ is defined, we furthermore study the space of paths in $M_{r, \lambda}=X_{r} \cap c^{-1}(-\infty, \lambda]$ and show how resulting path classes can be obtained. We evaluate our approach in 2, 3,4 , and 6 dimensions in simulation and using a Baxter robot.

\section{Motivation And Related Work}

For a robot to reason efficiently about trajectories within its own free configuration space $\mathcal{C}_{f}$, or about the motions of other human or robot agents in its environment, a suitable partitioning of continuously varying families of trajectories into a discrete set of equivalence classes is desirable.

Clustering trajectories is difficult in general since trajectories can have varying length and are not immediately representable as vectors in a vector space of fixed dimension as required by commonly used algorithms. Several approaches to the classification of trajectories, as reviewed in [33], are based on various approaches to measuring the dissimilarity between trajectories, such as the Hausdorff distance, edit distances and dynamic time warping. For the purpose of activity analysis, the work of [29] reviews trajectory clustering approaches based on various clustering algorithms and distance measures.

In robotics, the knowledge of classes of trajectories is beneficial for example in the learning by demonstration framework [8] where movement primitives of a robot's behavior are constructed from initial trajectory demonstrations provided by a human teacher. Equivalence classes of robot trajectories can furthermore be useful in order to reason about alternative trajectories when a subset of trajectories becomes invalid due 

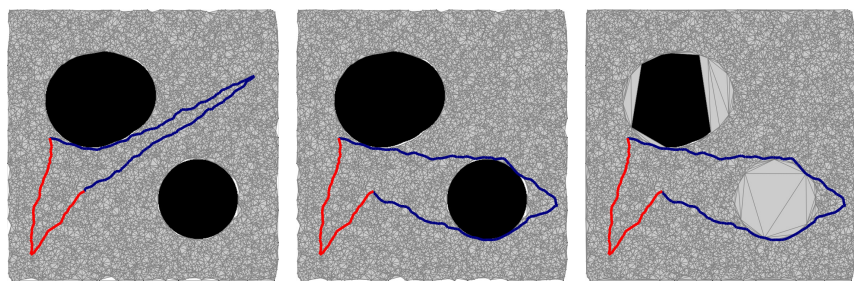

Fig. 1: We display a rectangular configuration space $\mathcal{C}$ of side-length 500 and with two obstacles (in black). Several trajectories are depicted in red and blue. The gray area displays an approximation of $\mathcal{C}_{f}$ by a Delaunay-Čech complex $D C_{r}(X)$ from 10000 samples $X \subset \mathcal{C}_{f}$ and with filtration value $r=11.07$ (left and middle figure) and $r=73.76$ (right figure). All of our figures are best viewed in color.

to changing environment conditions. The recent work [22] has demonstrated the usefulness of trajectory classes of local paths to improve the efficiency of a motion planning algorithm in particular. Purely topological approaches to the analysis of trajectories in $\mathcal{C}_{f}$ focus on notions of equivalence which do not depend on a metric. There, two paths $\alpha, \beta:[0,1] \rightarrow \mathcal{C}_{f}$ with $\alpha(0)=\beta(0), \alpha(1)=\beta(1)$ are called homotopy equivalent if $\alpha$ can continuously be deformed to $\beta$ in $\mathcal{C}_{f}$ while keeping the end-points fixed. We use Fig. 1 as a running example for illustration. Note that all figures in this paper are best viewed in color. For now, consider $\mathcal{C}_{f}$ as being approximated by the gray region, while the black regions correspond to obstacles. Several trajectories with identical start and end-points are depicted in red and blue. The blue trajectory in the leftmost figure is homotopy equivalent to the red trajectory, while the trajectories in the middle figure are not homotopy equivalent. Note that, while trajectories can have identical distance in $\mathbb{R}^{2}$ under e.g. the Hausdorff distance, they may or may not be homotopy equivalent. When the lower obstacle is removed in the right figure, the red and blue paths from the middle figure become homotopy equivalent, for example.

Topological concepts such as retractions and cell decompositions have played a key role in classical approaches to motion planning [23]. There, $\mathcal{C}_{f}$ is typically assumed to have a known algebraic or semi-algebraic structure. The visibility graph in 2D and retraction-based methods rely on constructing a graph using which motion planning is performed. The general roadmap method of [10] uses ideas closely related to Morse theory and projections to lower-dimensional spaces to obtain a complete motion planner for semi-algebraic sets. Similarly, the seminal work of [31] proceeds by constructing an exact cell decomposition by means of a cylindrical algebraic decomposition of $\mathcal{C}_{f}$. This is related to our approach since our simplicial complexes form a particular type of approximate cell decomposition. In [31], homology groups of $\mathcal{C}_{f}$ are computed by an exact cell decomposition $\mathcal{Z}$ and the general path planning problem is solved using $\mathcal{Z}$. These classical works have to the best of our knowledge however not considered the use of the first homology group of $\mathcal{C}_{f}$ for trajectory classification, and the focus has been on motion planning and not classification. An important difference to our work is the fact that we only assume the knowledge of potentially noisy point-samples from $\mathcal{C}_{f}$ using which we build a simplicial complex filtration rather than assuming a known description of $\mathcal{C}_{f}$ as a (semi-)algebraic set. Furthermore, our approach allows us to study the homotopy equivalence of paths within the neighborhood $X_{r}$ of a set of samples $X \subset \mathcal{C}_{f}$ more generally, e.g. when $X_{r}$ does not yield a reconstruction of the full space $\mathcal{C}_{f}$. In a more recent related work, [32] construct an approximate cell decomposition using a recursively refined decomposition of $\mathcal{C}_{f}$ into hypercubes to ensure a sufficiently fine reconstruction of $\mathcal{C}_{f}$. However, only the path-connectivity of this decomposition is then used for motion planning and homological properties are not further investigated.

Our work is also related to sampling-based algorithms constructing a graph $\Gamma$ from $X$ to answer questions about the path-connectivity of $\mathcal{C}_{f}$. RRTs and PRMs $[25,24,20]$, in particular, are examples of these which have attracted unabated interest since their invention $[26,27,19]$. The graph $\Gamma$ can be thought of as an approximation of $\mathcal{C}_{f}$ from $X$. The filtrations of simplicial complexes used in our work extend the concept of a graph to a multiscale approach which can recover more detailed information about $\mathcal{C}_{f}$. Such filtrations depending on a scale parameter $r$ have been used in topological data analysis (TDA) $[15,11,16]$ to study the persistent homology groups which we use here and which capture information about the topology of data at all scales simultaneously.

One of the advantages of the knowledge of homotopy classes is that a motion planning algorithm can utilize efficient replanning within each such class [9]. Since local variational or gradient based methods can continuously deform trajectories towards local optimality, it is advantageous to maintain a set of homotopy inequivalent trajectories each of which can then be optimized using these methods. Topological information about path classes hence allows us to incorporate non-trivial global information with these local methods.

Recent approaches which attempt to obtain equivalence classes of paths include the works $[18,17]$ on path deformation roadmaps where a graph-based representation to plan in the space of paths up to a class of continuous deformations is proposed. Recently, researchers have in particular investigated homotopy classes of trajectories in explicitly described spaces. Using the classical residue theorem of complex analysis [5] studied an application of homology classes to motion planning in 2D in the case where the obstacles in $\mathcal{C}$ can be contracted into representative and explicitly defined points. In [6], this was extended using electromagnetism theory and Ampère's law to the case of 3D. There, obstacles were assumed to be contracted into skeletons and then modeled as currentcarrying wires. Similarly to our work, the authors argue that homological information is useful and computationally favorable to more general homotopy invariants in robotics. In [7], a generalization to arbitrary dimension is proposed and an integration of differential 1-forms over cycles is shown to be sufficient to determine topological classes using the classical language of de Rham cohomology theory. In [21], motion planning in 2D with homology constraints is formulated as a mixed-integer quadratic program by endowing path segments with binary labels that identify their relation to the domain obstacles. A problem the above recent approaches suffer from 
is that they require an explicit description of the obstacles in the configuration space, e.g. in 2D as unions shapes each of which is contractible to a geometrically specified point $p \in \mathcal{C}-\mathcal{C}_{f}$. In many cases, such information is however not easily available for real robotic systems or too expensive to compute. We instead propose a data-driven, samplingbased approach to building a representation of $\mathcal{C}_{f}$ from which topological information about trajectories can be extracted.

\section{Theoretical BACKGROUND}

Filtrations and persistent homology provide a key tool to determine multiscale topological properties. We review some concepts from topological data analysis $[15,16]$.

\section{A. Delaunay-Čech Complexes}

A (abstract) $k$-simplex $\sigma$ is a set of $k+1$ elements, and we call $k$ the dimension of $\sigma$. A (abstract) simplicial complex $\mathcal{K}$ is a finite non-empty set of simplices such that if $\sigma \in \mathcal{K}$ and $\emptyset \neq \tau \subseteq \sigma \in \mathcal{K}$, then $\tau \in \mathcal{K}$. An element $\sigma \in \mathcal{K}$ is called a simplex of $\mathcal{K}$ and $\tau \subseteq \sigma$ is called a face of $\sigma$.

Consider a set of uniformly sampled points $X=$ $\left\{x_{1}, \ldots, x_{n}\right\} \subset Y$ from a subset $Y \subseteq \mathbb{R}^{d}$. The $r$ neighborhood $X_{r}=\bigcup_{i=1}^{n} \mathbb{B}_{r}\left(x_{i}\right)$, where $\mathbb{B}_{r}\left(x_{i}\right)=\left\{x \in \mathbb{R}^{d}\right.$ : $\left.\left\|x-x_{i}\right\| \leqslant r\right\}$ for $r \geqslant 0$, forms an interesting topological space. To compute the homology of $X_{r}$, we can represent $X_{r}$ by any simplicial complex $\mathcal{K}_{r}$ which is homotopy equivalent to $X_{r}$. The Cech complex $C_{r}(X)=\left\{\sigma \subseteq X: \cap_{x \in \sigma} \mathbb{B}_{r}(x) \neq \emptyset\right\}$ is an abstract such complex which however has no direct representation as a subset of $\mathbb{R}^{d}$. Given $X$, we can instead consider the complex $D(X)=\left\{\sigma \subseteq X: \cap_{x \in \sigma} V_{x} \neq \emptyset\right\}$ (the Delaunay triangulation of $X$ ) where $V_{x}$ denotes the Voronoi cell containing $x$. We consider $D(X)$ for points in $X$ which are in general position, which occurs with probability one and which can also be enforced by a small perturbation of $X$.

The Delaunay-Čech complex $D C_{r}(X)$, for $r \geqslant 0$, is the subcomplex of $D(X)$ defined by $D C_{r}(X)=\{\sigma \in$ $\left.D(X): \cap_{x \in \sigma} \mathbb{B}_{r}(x) \neq \emptyset\right\}$. The recent work [3] establishes that $D C_{r}(X)$ is homotopy equivalent to $X_{r}$, so that topological information about $X_{r}$ can be extracted from $D C_{r}(X)$ directly. We define $f: D(X) \rightarrow \mathbb{R}$ by $f(\sigma)=\min \left\{r: \cap_{x \in \sigma} \mathbb{B}_{r}(x) \neq\right.$ $\emptyset\}$, so that $D C_{r}(X)=f^{-1}((-\infty, r])$ and $D C_{r}(X)$ changes only at finitely many $r_{1}<\ldots<r_{m}$ which can be computed at all scales by determining $f(\sigma)$ for each simplex $\sigma \in D(X)$. Every $k$-simplex $\sigma=\left\{v_{0}, \ldots, v_{k}\right\} \in D C_{r}(X)$ corresponds to the geometric simplex given by the convex hull $\operatorname{Conv}(\sigma)$, so that 0 -simplices are points, 1 -simplices are edges, and 2simplices are triangles. Fig. 1 and 2 illustrate examples of $D C_{r}(X)$ in $\mathbb{R}^{2}$. Note that, instead of $D C_{r}(X)$, we could also have considered the Alpha complexes $A_{r}(X)$ of [14] since $A_{r}(X)$ is also homotopy equivalent to $X_{r}$ and furthermore $A_{r}(X) \subseteq D C_{r}(X), A_{\infty}(X)=D C_{\infty}(X)=D(X)$. An advantage of $D C_{r}(X)$ over $A_{r}(X)$ in our application is that we can compute the filtration values for the 2-skeleton of $D C_{r}(X)$ consisting only of simplices of $D C_{r}(X)$ up to dimension 2 directly without having to first compute the filtration values for the higher skeleta.

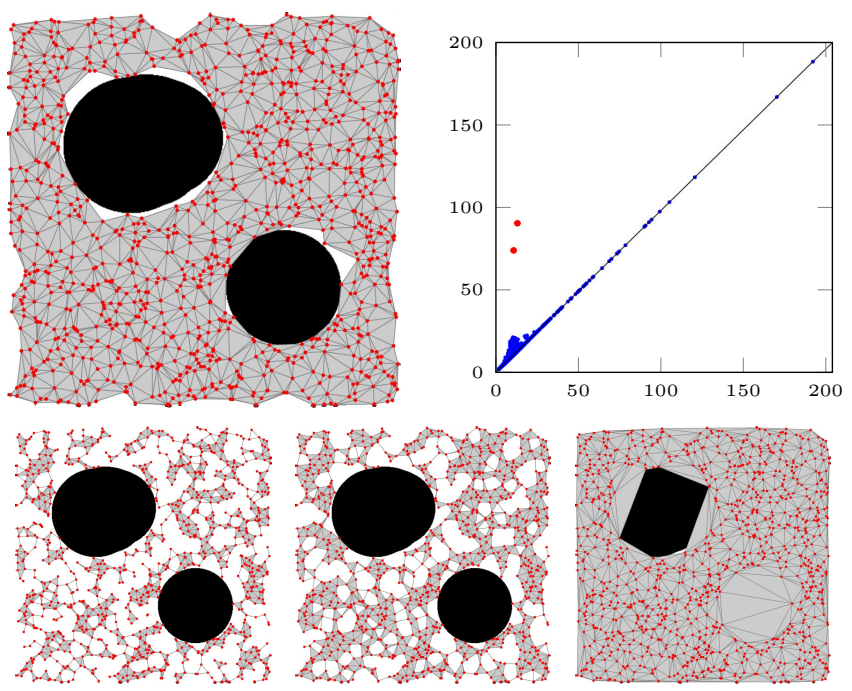

Fig. 2: A reconstruction of $\mathcal{C}_{f}$ from 1000 samples (red points) on a square of side-length $500 . D C_{25}(X)$ is displayed in the top-left yielding a good approximation to $\mathcal{C}_{f}$. The first persistence diagram for $D C_{r}(X)$ is shown in the top-right. The two marked red points $p_{1}=(10.58,74.0)$, $p_{2}=(12.97,90.38)$ with large persistence correspond to the birth and death filtration of the two holes in $\mathcal{C}_{f}$. The bottom row displays $D C_{10.58}(X)$, $D C_{12.97}(X)$ and $D C_{74.0}(X)$ which correspond to the birth of the smaller and larger hole (the first time they are enclosed by edges), and finally to the death filtration value of the smaller hole (the hole is covered at $r=74.0$ ).

\section{B. Filtrations and Homology}

We now review the details of simplicial homology over $\mathbb{Z}_{2}=\{0,1\}$ for a geometric simplicial complex $\mathcal{K}$ in $\mathbb{R}^{d}$. The example to keep in mind is the case $\mathcal{K}=D C_{r}(X)$ for a fixed $r \geqslant 0$. We then extend the discussion to a collection of simplicial subcomplexes of a simplicial complex $\mathcal{K}$ defined by $\mathcal{K}_{r}=f^{-1}((-\infty, r])$, where $f: \mathcal{K} \rightarrow \mathbb{R}$ satisfies $f(\tau) \leqslant f(\sigma)$ whenever $\tau \subseteq \sigma \in \mathcal{K}$. Then $\mathcal{K}_{r} \subseteq \mathcal{K}_{r^{\prime}}$ whenever $r \leqslant r^{\prime}$ yielding a filtration of simplicial complexes. From the preceding discussion, we observe that $\mathcal{K}_{r}=D C_{r}(X)$, with $\mathcal{K}_{\infty}=D(X)$, yields a filtration as $r$ varies.

A $p$-chain $c$ is a formal sum $c=\sum_{i=1}^{k} \lambda_{i} \sigma_{i}$ of $p$-simplices $\left\{\sigma_{i}\right\}_{i=1}^{k} \subset \mathcal{K}$ with $\lambda_{i} \in \mathbb{Z}_{2}$ and $C_{p}(\mathcal{K})$ denotes the vector space of all $p$-chains. In particular, 1-chains are finite sets of edges and 2-chains are finite sets of triangles. For every $p$ simplex $\sigma$ let $\partial \sigma$ be the $p-1$-chain formed by the formal sum of all $p-1$ dimensional faces of $\sigma$ corresponding to its boundary. $\partial$ extends to a linear map $\partial: C_{p}(\mathcal{K}) \rightarrow C_{p-1}(\mathcal{K})$. A chain $c \in C_{p}(\mathcal{K})$ such that $c=\partial \omega$ for some $\omega \in C_{p+1}(\mathcal{K})$ is called a $p$-boundary, and we call $c$ a $p$-cycle if $\partial c=0$. The set of $p$-boundaries and $p$-cycles is denoted by $B_{p}(\mathcal{K})$ and $Z_{p}(\mathcal{K})$ respectively and $B_{p}(\mathcal{K}) \subseteq Z_{p}(\mathcal{K})$ since $\partial \partial=0$. The quotient vector space $H_{p}(\mathcal{K})=Z_{p}(\mathcal{K}) / B_{p}(\mathcal{K})$ is called the $p^{\text {th }}$ homology group of $\mathcal{K}$ and $b_{p}(\mathcal{K})=\operatorname{dim}\left(H_{p}(\mathcal{K})\right)$ is called the $p^{\text {th }}$ Betti-number. $b_{0}(\mathcal{K})$ is equal to the number of connected components of $\mathcal{K}$. We denote the equivalence class of a $p$-cycle $c$ in $H_{p}(\mathcal{K})$ by $[c]$. To understand what a 1 -cycle is, observe that the boundary of a 1-simplex (i.e. an edge) consists of the two vertices (i.e. 0-simplices) connected by the 1-simplex. Similarly, the boundary of a 1-chain (i.e. a collection of edges) in $\mathcal{K}$ consists of the sum of boundaries of the 1 -simplices in the chain counted modulo $\mathbb{Z}_{2}$. In particular, 
any closed edge-path $\gamma$ in $\mathcal{K}$ is a 1-cycle which is trivial in $H_{1}(\mathcal{K})$ if it does arise as the boundary of a 2-chain (i.e. a collection of triangles) and two 1-cycles $\gamma, \gamma^{\prime}$ are equivalent if their difference (equivalently their sum modulo $\mathbb{Z}_{2}$ ) is the boundary of a 2-chain. In Fig. 1, the union $c$ of the red and blue paths are 1-cycles in $D C_{r}(X)$. In the leftmost and rightmost figure $[c]=0$, while $[c] \neq 0$ in the middle figure for the indicated filtration values $r$. A basis for $H_{1}\left(D C_{r}(X)\right)$ for the leftmost figure can be provided by two equivalence classes of cycles $[c],\left[c^{\prime}\right]$, for example where $c$ loops around the larger hole once and $c^{\prime}$ loops around the smaller hole once. However, the choice of representative cycles $c, c^{\prime}$ is not unique. In these examples, $b_{1}(\mathcal{K})=\operatorname{dim}\left(H_{1}(\mathcal{K})\right)$ hence measures the number of enclosed voids in $\mathcal{K}=D C_{r}(X)$. Since $H_{1}\left(D C_{r}(X)\right)$ changes with the filtration value $r$, we now recall how to study the changes in homology using persistent homology [15].

a) Persistent Homology: For a filtration of simplicial complexes, where $f: \mathcal{K} \rightarrow \mathbb{R}, \mathcal{K}$ is a finite simplicial complex, and $\mathcal{K}_{r}=f^{-1}((-\infty, r])$, we denote the finitely many filtration values at which $\mathcal{K}_{r}$ changes by $r_{1}<\ldots<r_{m}$. The inclusion $\alpha_{i}^{j}: \mathcal{K}_{r_{i}} \rightarrow \mathcal{K}_{r_{j}}$, for $i \leqslant j$, induces a linear map $h_{i}^{j}: H_{p}\left(\mathcal{K}_{r_{i}}\right) \rightarrow H_{p}\left(\mathcal{K}_{r_{j}}\right)$. We say that a homology class $\alpha \in H_{p}\left(\mathcal{K}_{r_{i}}\right)$ is born at $r_{i}$ if $\alpha \notin i m\left(h_{i-1}^{i}\right)$. A class $\alpha \in$ $H_{p}\left(\mathcal{K}_{r_{i}}\right)$ born at $r_{i}$ is said to die at $r_{j}$ if $h_{i}^{j-1}(\alpha) \notin i m\left(h_{i-1}^{j-1}\right)$, but $h_{i}^{j}(\alpha) \in i m\left(h_{i-1}^{j}\right)$. The difference $r_{j}-r_{i}$ is called the persistence of $\alpha$ : it measures how long a homological feature survives in the filtration. Classes born at $r_{i}$ which do not die are associated to $\left(r_{i}, \infty\right)$ and are called essential, the remaining classes are called inessential. Similarly, if a cycle represents an essential (inessential) class, we call the cycle essential (inessential). For $i \leqslant j$, the $p$-th persistent homology group is defined as $H_{p}^{i, j}=Z_{p}\left(\mathcal{K}_{r_{i}}\right) /\left(B_{p}\left(\mathcal{K}_{r_{j}}\right) \cap Z_{p}\left(\mathcal{K}_{r_{i}}\right)\right)$. Non-trivial elements of $H_{p}^{i, j}$ correspond to equivalence classes of $p$-cycles born at or before $r_{i}$ and which persist, i.e. do not die in the filtration for $r \in\left[r_{i}, r_{j}\right)$. For $i=j$, this recovers the usual notion of homology $H_{p}^{i, i}=H_{p}\left(\mathcal{K}_{r_{i}}\right)=Z_{p}\left(\mathcal{K}_{r_{i}}\right) / B_{p}\left(\mathcal{K}_{r_{i}}\right)$. A graphical representation is obtained by the $p$-th persistence diagram which associates $\left(r_{i}, r_{j}\right)$ to classes born at $r_{i}$ and dying at $r_{j}$ and $\left(r_{i}, \infty\right)$ to essential classes born at $r_{i}$ (with multiplicity). The number of points in $\left(-\infty, r_{i}\right] \times\left(r_{j}, \infty\right]$ equals $\operatorname{dim}\left(H_{p}^{i, j}\right)$ and the vertical distance of a point to the diagonal indicates how long the feature persists (see [15]).

In Fig. 2, we display the diagram for $p=1$ and $D C_{r}(X)$. Observe that the two obstacles correspond to the two red points in the diagram which are far from the diagonal. The remaining points correspond to holes which are due to noise and which do not persist for a large filtration interval. Note also that, for $p=0$, the persistence diagram measures the merging of connected components of $D C_{r}(X)$ as $r$ is increased [15].

b) Computation via matrix reduction: To compute the persistence diagrams of a filtration $\mathcal{K}_{r_{1}} \subset \mathcal{K}_{r_{2}} \subset \ldots \subset \mathcal{K}_{r_{m}}$, it is convenient to refine the filtration as follows: we pick an ordering $\sigma_{1}, \ldots, \sigma_{n}$ of the simplices of $\mathcal{K}_{r_{m}}$ such that, for all $i \in\{1, \ldots, n\}, K_{i}=\cup_{l=1}^{i} \sigma_{l}$ is a simplicial complex and there exist indices $0 \leqslant i_{1}<i_{2}<\ldots<i_{m}=n$ such that
$K_{i_{j}}=\mathcal{K}_{r_{j}}$. Such a simplexwise filtration can be obtained by inserting simplices in $\mathcal{K}_{r_{i}}$ before simplices in $\mathcal{K}_{r_{j}}$ if $i<j$ and by inserting the faces $\tau \subset \sigma$ of any simplex $\sigma$ before inserting $\sigma$ itself [15].

Let $K=\bigcup_{i=1}^{n} \sigma_{i}$ be such a simplexwise filtration. The boundary operator $\partial: \oplus_{p=0}^{d} C_{p}(K) \rightarrow \oplus_{p=0}^{d} C_{p}(K)$ is a linear map which we express in the ordered basis $\sigma_{1}, \ldots, \sigma_{n}$ yielding an $n \times n$ matrix $D$ with $\mathbb{Z}_{2}$ entries. For a matrix $M$, we denote by $M_{j}$ the $j^{\text {th }}$ column and by $M_{i j}$ the $(i, j)$-entry. Note that $D$ is upper triangular and $D_{i j}=1$ if $\sigma_{i}$ is a codimension 1 face of $\sigma_{j}$. We let $\operatorname{low}\left(M_{j}\right)=\max \left\{i: M_{i j} \neq 0\right\}$ if $M_{j} \neq 0$ and $\operatorname{low}\left(M_{j}\right)$ is undefined otherwise. A left-toright column addition $M_{j} \leftarrow M_{j}+M_{i}, i<j$ is called reducing if it decreases low $\left(M_{j}\right)$ and $M$ is called reduced if no reducing left-to-right column addition can be performed on any of its columns. The standard persistence algorithm [16] applies left-to-right column additions to $D$ until $D$ is reduced, yielding a reduced matrix $R$. We can keep track of these additions by initializing the algorithm with $R=D$, $V=I_{n}$, so that $R=D V$. For each left-to-right column addition $R_{j} \leftarrow R_{j}+R_{i}$ for $i<j$, we perform the column addition $V_{j} \leftarrow V_{j}+V_{i}$. This algorithm terminates when $R$ is reduced and we have $R=D V$, where $V$ is the matrix relating $R$ to its unreduced version $D$. One defines [12] $P=\left\{(i, j): R_{j} \neq 0\right.$ and $\left.i=\operatorname{low}\left(R_{j}\right)\right\}, E=\left\{i: R_{i}=\right.$ 0 and $\operatorname{low}\left(R_{j}\right) \neq i$ for all $\left.j \in\{1, \ldots, n\}\right\}$. Returning to $\mathcal{K}_{r}=f^{-1}((-\infty, r])$, each $(i, j) \in P$ with $\operatorname{dim}\left(\sigma_{i}\right)=p$ corresponds to $\left(f\left(\sigma_{i}\right), f\left(\sigma_{j}\right)\right)$ in the $p$-th persistence diagram and is generated by the $p$-cycle $R_{j}$ which dies with the introduction of the simplex $\sigma_{j}$. Similarly, each $i \in E$ with $\operatorname{dim}\left(\sigma_{i}\right)=p$ corresponds to $\left(f\left(\sigma_{i}\right), \infty\right)$ and the $p$-cycle $V_{i}$ which is still alive in the final filtration $K_{n}=\mathcal{K}_{r_{m}}$. Note that the cycles $V_{i}$, $R_{j}$ are not canonical, but the persistence diagrams determine the ranks of all persistent homology groups.

c) $H_{1}(Y)$ and homotopy classes of trajectories: The final piece of background work we require is the connection between the first homology group and homotopy classes of paths in a topological space $Y$. The obvious case to keep in mind is $Y=\mathcal{C}_{f} \subset \mathbb{R}^{d}$. Recall that the first fundamental group $\pi_{1}\left(Y, x_{0}\right)$ [16] is a well-known group whose elements consist of equivalence classes of closed continuous curves through $x_{0} \in Y$ and lying entirely in $Y$. Two closed paths $\alpha, \beta:[0,1] \rightarrow Y$ through $x_{0}$ lie in the same equivalence class if there exists a homotopy (i.e. a continuous deformation) between them which is constant at the base-point $x_{0}$. When $Y$ is path-connected, $\pi_{1}\left(Y, x_{0}\right)$ is independent of the chosen base-point $x_{0}$ and hence often denoted simply by $\pi_{1}(Y)$. Furthermore, if the spaces $Y, Y^{\prime}$ are homotopy equivalent spaces, $\pi_{1}(Y)$ and $\pi_{1}\left(Y^{\prime}\right)$ are isomorphic as groups. Two paths $\gamma_{1}, \gamma_{2}$ in $Y$ with the same start point $x$ and end point $y$ can be deformed into each other via a homotopy if the closed curve $\gamma$ following $\gamma_{1}$ from $x$ to $y$ and then $\gamma_{2}$ from $y$ to $x$ is trivial in $\pi_{1}(Y)$. Hence, $\pi_{1}(Y)$ is a natural group to consider for the purpose of trajectory classification. Unfortunately, to the best of our knowledge, no sufficiently efficient method for general configuration spaces exists to compute the group structure of 
$\pi_{1}(Y)$ which can be complicated and non-commutative. To extract topological information about homotopy classes, we can turn to the first singular homology group $H_{1}(Y)$ with binary $\mathbb{Z}_{2}=\{0,1\}$ coefficients, yielding a vector space which can be explicitly computed via simplicial homology when $Y$ is homotopy equivalent to a simplicial complex $\mathcal{K}$. The closed curve $\gamma$ can be represented explicitly as a 1 -cycle in a sufficiently fine subdivision of $\mathcal{K}$ when a deformation retraction from $Y$ to $\mathcal{K}$ is computable, and $\gamma$ then corresponds to a vector $[\gamma]$ in $H_{1}(Y) \cong H_{1}(\mathcal{K})$. Finally, $[\gamma] \neq 0$ implies that $\gamma_{1}$ and $\gamma_{2}$ are not homotopy equivalent, allowing us to discern homotopy classes of continuous paths. Note however that homology is a weaker concept than homotopy, so $[\gamma]=0 \in H_{1}(Y)$ does not imply that $\gamma_{1}$ and $\gamma_{2}$ are homotopy equivalent. To gain somewhat more granularity, one can further replace $\mathbb{Z}_{2}$ coefficients for example with $\mathbb{Z}_{p}$ coefficients for a large prime $p$. In this work we choose $\mathbb{Z}_{2}$ coefficients due to their computational advantages for large simplicial complexes.

\section{Methodology}

We consider a configuration space $\mathcal{C} \subset \mathbb{R}^{d}$ and the set $\mathcal{C}_{f} \subseteq$ $\mathcal{C}$ of collision-free configurations. We do not assume that we have an explicit description of $\mathcal{C}_{f}$ or $\mathcal{C}$ available, and we would like to study homotopy classes of a set of trajectories $T=$ $\left\{\gamma_{1}, \ldots, \gamma_{k}\right\} \subset \mathcal{C}_{f}$ with a fixed starting point $x \in \mathcal{C}_{f}$ and end point $y \in \mathcal{C}_{f}$. In order to classify the trajectories, we shall exploit the connection between homotopy classes and the first homology group which we just discussed. We now consider two multiscale settings:

1) $X$ is a sufficiently dense sample: We assume that $X=\left\{x_{1}, \ldots, x_{n}\right\} \subset \mathcal{C}_{f}$ yields a sufficiently dense sample, for example sampled via rejection sampling from the uniform distribution on $\mathcal{C}$, or via a randomized exploration of the configuration space. We can then ask about a likely approximation of $\mathcal{C}_{f}$ from $X$. Our working hypothesis is that the family of spaces $\left\{X_{r}=\bigcup_{x \in X} \mathbb{B}_{r}(x): r>0\right\}$ contain good such estimates. If $X$ was sampled uniformly and $\mathcal{C}_{f}$ is a smooth compact submanifold $M \subset \mathbb{R}^{d}$, this intuition is in fact well-founded due to the reconstruction theorem of [30] which guarantees that, for a sufficiently dense sample set, $X_{r}$ deformation retracts to the manifold $M$ for appropriately chosen $r$. Using the previously introduced Delaunay-Čech complex and the fact that $D C_{r}(X)$ is homotopy equivalent to $X_{r}$ [3], we will then compute homological information about $X_{r}$ from $D C_{r}(X)$.

2) $X \subset T$ : We assume only the availability of the trajectories $T$. We then discretize each trajectory $\gamma_{i}$ as a piecewise linear curve and use the vertex positions of all the piecewise linear segments in $T$ as our sample set $X$. We study the homotopy classes of these trajectories within the topological spaces $X_{r}$ which constitute an approximation of the $r$-neighborhoods around $T$. This then allows us to classify trajectories within $X_{r}$. In this framework, holes can arise either due to obstacles in the configuration space (as in the dense case), or due to the distribution of the trajectories in $\mathcal{C}_{f}$. We consider applications of this case in our experiments with a Baxter robot.

For a sample set $X$, let $R$ be the minimal $r>0$ such that $\gamma_{i} \subset X_{r}$ for all $i \in\{1, \ldots, k\}$. Our approach in both cases above will now be to study the homotopy classes of these paths in the topological spaces $X_{r} \simeq D C_{r}(X)$, for $r \geqslant R$.

\section{A. Trajectory Discretization}

In order to compute properties of a trajectory $\gamma:[0,1] \rightarrow$ $\mathcal{C}_{f}$, we first need to represent $\gamma$ by a homotopy equivalent path of edges (i.e. 1-simplices) in $D C_{R}(X)$. A fast heuristic procedure for this is to consider $v_{i}=\gamma(i / N)$, for some large $N \in \mathbb{N}$, to map $v_{i}$ to a closest 0 -simplex $v_{i}^{\prime} \in D C_{R}(X)$ and to then replace the path segment between $v_{i}, v_{i+1}$ by a shortest edge-path between $v_{i}^{\prime}$ and $v_{i+1}^{\prime}$. Alternatively, one can attempt to construct an explicit deformation retraction from $X_{R}$ to $D C_{R}(X)$ mapping $\gamma$ first to a path contained in $D C_{R}(X)$ and then approximating $\gamma$ by a homotopy equivalent sequence of 1-simplices on a sufficiently fine subdivision of $D C_{R}(X)$. The Alpha complexes $A_{r}(X)$ of [14] are subcomplexes of $D C_{r}(X)$ for all $r>0$ which are also homotopy equivalent to $X_{r}$ and onto which an explicit such deformation retraction from $X_{r}$ has been described in [14], for example. While the study of efficient and theoretically sound homotopy equivalent trajectory discretizations should be explored further, we will instead focus on the classification problem here, assuming that each trajectory has been discretized as a path of edges in $D C_{R}(X)$.

\section{B. Homological Trajectory Classification}

Consider a set of edge-paths $\left\{\alpha_{0}, \ldots, \alpha_{m}\right\}$ in $D C_{R}(X)$ starting and ending at 0 -simplices $s, t \in D C_{R}(X)$ respectively. We consider the 1 -cycle $c_{\alpha_{0}}\left(\alpha_{u}\right) \stackrel{\text { def }}{=} \alpha_{0}+\alpha_{u} \in$ $Z_{1}\left(D C_{R}(X)\right)$. Now $\left[c_{\alpha_{0}}\left(\alpha_{u}\right)\right] \neq\left[c_{\alpha_{0}}\left(\alpha_{w}\right)\right] \in H_{1}^{i, j}(D C(X))$ implies $\left[\alpha_{u}+\alpha_{w}\right] \neq 0$, so that $\alpha_{u}, \alpha_{w}$ are not homotopy equivalent in $D C_{r}(X), R \leqslant r_{i} \leqslant r<r_{j}$, where $r_{1}<\ldots<r_{m}$ denote the critical filtration values at which $D C_{r}(X)$ changes. We hence have trajectory classes $\left\{\left[c_{\alpha_{0}}\left(\alpha_{0}\right)\right], \ldots,\left[c_{\alpha_{0}}\left(\alpha_{m}\right)\right]\right\} \in H_{1}^{i, j}$ and the class membership can be computed once we have determined a basis for $H_{1}^{i, j}$. Note that $\alpha_{0}$ corresponds to the zero vector $0=\left[c_{\alpha_{0}}\left(\alpha_{0}\right)\right]$ and there can be up to $2^{k}$ trajectory classes for fixed $s, t$ and $i, j$ when $\operatorname{dim}\left(H_{1}^{i, j}\right)=k$. We can now compute a basis for $H_{1}^{i, j}$ :

Lemma. Let $K_{1} \subset \ldots \subset K_{n}$ be a simplexwise filtration of simplicial complexes, let $R=D V$ denote the reduced boundary matrix after applying the left-to-right reduction algorithm, and let $E_{p} \subseteq E, P_{p} \subseteq P$ denote those elements corresponding to $p$-cycles only. For $1 \leqslant i \leqslant n$, a basis of $Z_{p}\left(K_{i}\right)$ is given by $S^{i}=\left\{R_{t}:(s, t) \in P_{p}, s \leqslant i\right\} \cup\left\{V_{s}: s \in E_{p}, s \leqslant i\right\}$, and, for $1 \leqslant i \leqslant j \leqslant n$, the image of the set

$T^{i, j}=\left\{R_{t}:(s, t) \in P_{p}, s \leqslant i, t>j\right\} \cup\left\{V_{s}: s \in E_{p}, s \leqslant i\right\}$

in $H_{p}^{i, j}=Z_{p}\left(K_{i}\right) /\left(B_{p}\left(K_{j}\right) \cap Z_{p}\left(K_{i}\right)\right)$ forms a basis of $H_{p}^{i, j}$. Finally $\# E_{p}=\operatorname{dim}\left(H_{p}\left(K_{n}\right)\right)$.

Proof: This follows from the reduction algorithm [16]. 
In order to classify $\left\{\alpha_{0}, \ldots, \alpha_{m}\right\}$, we first select a simplexwise refinement $\left\{K_{i}\right\}_{i=1}^{n}$ of the filtration given by $D C_{r}(X)$, $r \geqslant 0$. Next, we compute the $\mathbb{Z}_{2}$ coordinates of $c_{\alpha_{0}}\left(\alpha_{u}\right)$ for $0 \leqslant u \leqslant m$ in the basis $S^{n}$ once. To classify trajectories at a scale given by the filtration value $r_{i}=f(\sigma)$, we simply look up the binary coordinates of $c_{\alpha_{0}}\left(\alpha_{u}\right)$ restricted to the basis elements $T^{i, i} \subseteq S^{n}$. Similarly, we can check if two trajectories $\alpha_{u}, \alpha_{w}$ are homotopy inequivalent for all $r_{i} \leqslant r<r_{j}$ by looking up whether the coordinates of $c_{\alpha_{0}}\left(\alpha_{u}\right)$ and $c_{\alpha_{0}}\left(\alpha_{w}\right)$ differ in the basis $T^{i, j} \subseteq S^{n}$.

Note now that $D C_{r}(X)=D(X)$ for sufficiently large $r$, where $D(X)$ denotes the full Delaunay triangulation, and $H_{1}(D(X))=\{0\}$ since $D(X)$ is contractible. Hence $E_{1}$ is empty implying that we do not need to keep track of the matrix $V$ to determine a basis of $H_{1}^{i, j}$. This is important since, in our experiments, these matrices have millions of columns and $R$ is typically very sparse and of low rank, while $V$ has full rank. Since low is injective on the set $S^{n}$, we order elements of $S^{n}$ (for $p=1$ ) by their low value and we store $l o w^{-1}=l$ as a map such that $l(k)$ is that element $s \in S^{n}$ with $\operatorname{low}(s)=k$. For any cycle $c \in Z_{1}\left(K_{i}\right)$, we can then trivially solve for the coefficients in the basis $S^{n}$ by iterating $c \leftarrow c+l(\operatorname{low}(c))$. Each iteration reduces $l o w(c)$ until we arrive at the zero vector. In the ordered basis $S^{n}, c$ then has non-zero coefficients $F(c) \in \mathbb{Z}_{2}^{\# S^{n}}$ exactly at those basis elements $s \in S^{n}$ for which $\operatorname{low}(s)=\operatorname{low}(c)$ during the execution of the above loop. Again, $n$ can be very large (millions), but the vector $F(c)$ is in our experiments very sparse so that the algorithm does not exhibit its worst cast $O\left(n^{2}\right)$ computation time. We call $F\left(c_{\alpha_{0}}\left(\alpha_{u}\right)\right) \in \mathbb{Z}_{2}^{\# S^{n}}$ the persistent cycle coordinates of $\alpha_{u}$ with respect to $\alpha_{0}$.

If we want to determine a trajectory class at scales corresponding to filtration values $r_{i}<r_{j}$, we select the coordinates $F^{i, j}\left(c_{\alpha_{0}}\left(\alpha_{u}\right)\right)$ of $F\left(c_{\alpha_{0}}\left(\alpha_{u}\right)\right)$ corresponding to the basis $T^{i, j}$. Two trajectories $\alpha_{u}, \alpha_{w}$ are then not homotopy equivalent if $F^{i, j}\left(c_{\alpha_{0}}\left(\alpha_{u}\right)\right) \neq F^{i, j}\left(c_{\alpha_{0}}\left(\alpha_{w}\right)\right.$. Each non-zero coordinate of $F\left(c_{\alpha_{0}}\left(\alpha_{u}\right)\right)$ corresponds to a column $R_{t}$ of $R$ which has a death filtration value $f\left(\sigma_{t}\right)$. At filtration value $r$, only those non-zero coordinates that have been born and have not died yet contribute to the classification of cycles. We hence obtain an agglomerative clustering of trajectories lying in a common $D C_{R}(X)$ as we increase the filtration value $r \geqslant R$. Finally, at $r_{m}, D C_{r_{m}}(X)=D C_{\infty}(X)=\operatorname{Conv}(X)$ and all trajectories then lie in the same class.

Illustration: Consider Fig. 1. The red trajectory corresponds to $\alpha_{0}$ and the two blue trajectories in the left and middle figure represent $\alpha_{1}, \alpha_{2}$ respectively, and all trajectories lie in $D C_{r_{i}}(X), r_{i}=11.07$. We have $\left[c_{\alpha_{0}}\left(\alpha_{0}\right)\right]=\left[c_{\alpha_{0}}\left(\alpha_{1}\right)\right]=$ $0 \in H_{1}^{a, b}$ for all $i \leqslant a \leqslant b$, but $\left[c_{\alpha_{0}}\left(\alpha_{2}\right)\right] \neq 0 \in H_{1}^{a, b}$, for $i \leqslant a \leqslant b \leqslant j$, where $r_{j}=73.76$ is the critical filtration value at which the hole surrounded by $\alpha_{0}, \alpha_{2}$ gets filled in.

\section{Filtrations with Cost Functions}

Suppose now that we have sampled $\mathcal{C}_{f}$ sufficiently densely and that $D C_{R}(X)$, for some fixed $R$, provides a good approximation of $\mathcal{C}_{f}$. Consider a cost-function $c: \mathcal{C}_{f} \rightarrow \mathbb{R}$. Our aim

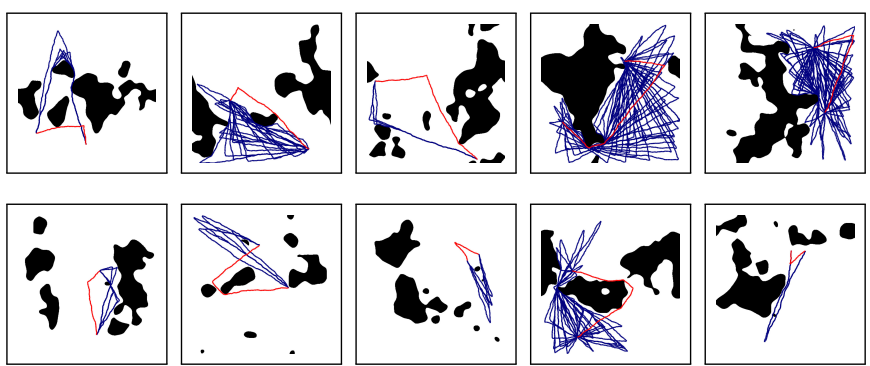

Fig. 3: We display example worlds and examples of paths which were determined to lie in a single class (in blue) at filtration value $r_{2} . D C_{r_{2}}(X)$ was constructed from 100000 samples and the classes are computed using the indicated red trajectories corresponding to $\alpha_{0}$.

now is not only to classify trajectories in the space $\mathcal{C}_{f}$, but to take into account a threshold for the cost function. Given $c$, we define the cost $\hat{c}$ of a $k$-simplex $\sigma=\left\{v_{0}, \ldots, v_{k}\right\} \in D C_{R}(X)$ to be $\hat{c}(\sigma)=\max \left(c\left(v_{0}\right), \ldots, c\left(v_{k}\right)\right)$. Then $\hat{c}$ satisfies $\hat{c}(\tau) \leqslant$ $\hat{c}(\sigma)$ whenever $\tau \subseteq \sigma$. In order to apply our algorithm without having to keep track of the potentially non-sparse matrix $V$, we furthermore let $\hat{c}(\sigma)=\max \left(c\left(v_{0}\right), \ldots, c\left(v_{k}\right)\right)+C$ for any $k$-simplex $\sigma \in D(X)-D C_{R}(X)$ and for $C$ larger than the cost of any $v \in X$. Then $L_{R, \lambda}=\hat{c}^{-1}((-\infty, \lambda])$ yields a filtration as $\lambda$ varies and $L_{R, \lambda} \subseteq D C_{R}(X)$ for $\lambda<C$ and $L_{R, \infty}=D(X)$, ensuring $H_{1}\left(L_{R, \infty}\right)=0$. We think of $L_{R, \lambda}$ as an approximation to $M_{R, \lambda}=X_{R} \cap c^{-1}((-\infty, \lambda])$ for $\lambda<C$.

\section{EXPERIMENTS}

Our experiments were performed on an Intel Core i7 laptop with 8GB of RAM. We present only the computation times of core algorithms and disregard the time required to initially load data into memory. We used the matrix and binary tree column vector data structure of the PHAT library [4] to efficiently manipulate large boundary matrices. Instead of working with the full simplicial complex $D C_{r}(X)$, we extracted the 2skeleton $D C_{r}^{2}(X)$ from the Delaunay triangulation $D(X)$. The 2-skeleton is sufficient for our purposes since $H_{1}^{i, j}\left(D C_{r}(X)\right)$ does not depend on higher dimensional simplices. We reduced only the submatrix of the boundary matrix corresponding to the first homology group. $D(X)$ was computed with CGAL [1] for all but our Baxter experiments where we used QHull [2] which was faster in higher dimensions. A supplementary video can be found at http://www.csc.kth.se/ fpokorny.

Trajectory classification in 2D: We generated the set of $2 \mathrm{D}$ worlds $W_{1}, \ldots, W_{10}$ displayed in Fig 3 and of size $512 \times 512$ by sampling Gaussian Random Fields and defining those regions above a threshold to be obstacles. From the resulting free space $\mathcal{C}_{f}$, we sampled $N \in$ $\{1000,10000,100000,1000000\}$ uniform samples. We computed the Delaunay-Čech filtration for all examples and recorded the computation times of the Delaunay triangulation, for the construction of the filtration, as well as the time required to reduce the boundary matrix $D$ to its reduced form $R$. The Delaunay triangulation took $1 \mathrm{~ms}, 2 \mathrm{~ms}, 76 \mathrm{~ms}, 810 \mathrm{~ms}$, the construction of the filtration took $11 \mathrm{~ms}, 31 \mathrm{~ms}, 278 \mathrm{~ms}$ and $3.27 \mathrm{~s}$ and the reduction of the boundary matrix took $14 \mathrm{~ms}$, $13 \mathrm{~ms}, 76 \mathrm{~ms}, 981 \mathrm{~ms}$ on average as the sample size increased. 

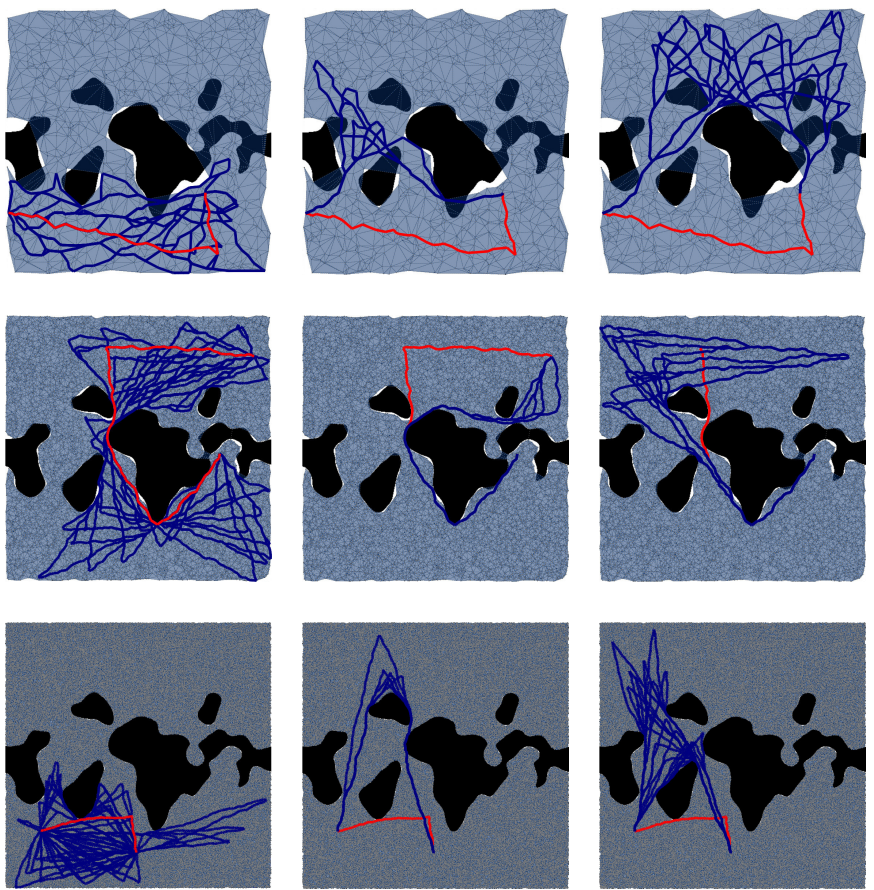

Fig. 4: We display the example world $W_{1}$ with $D C_{r_{2}}(X)$ for 1000,10000 and 100000 sample points per row. In each column, we plot paths $\alpha_{1}, \ldots, \alpha_{s}$ (in blue) which belong to a fixed trajectory class at filtration value $r_{2}$. The fixed reference path $\alpha_{0}$ is plotted in red. As expected, we can clearly see that two paths in different classes also lie in different homotopy classes. In our experiments, paths within a class are furthermore homotopy equivalent in $D C_{r_{2}}(X)$, but the quality of the approximation $D C_{r_{2}}(X) \simeq \mathcal{C}_{f}$ is only sufficient for 10000 or more sample points as can be seen in the right figure in the first row. There, some 2-simplices (triangles) cover the thin obstacle region to the right.

We investigated the filtration $D C_{r}(X)$ at various thresholds. At a filtration value of $r_{1}=25 \sqrt{1000 / N}$, we found that $\mathcal{C}_{f}$ was conservatively covered, while at $r_{2}=35 \sqrt{1000 / N}$, the space was well covered with a minimum number of holes in collision free areas. In order to investigate interesting path classes, we generated a set of 1000 paths per world and sample setting as follows: In 10 trials, we selected two sample points $v_{1}, v_{2}$ at random and, for each such setting, we selected another 100 random waypoints $w_{1}, \ldots, w_{100}$ from the sampled point-cloud. We determined shortest edge-path from $v_{1}$ to $w_{i}$ and then to $v_{2}$ utilizing Dijkstra's algorithm on the 1-skeleton graph of $D C_{r_{1}}(X)$. The computation times for the persistent cycle coordinates for these paths were $1.8 \mathrm{~ms}, 10 \mathrm{~ms}, 115 \mathrm{~ms}$ and $1.75 \mathrm{~s}$ for a batch of 100 query paths and for the respective sample sizes on average. These encouraging timings suggest that our framework could be used as a classification 'black box' e.g. for continuous trajectory optimization engines.

Trajectory classification in 4D: We consider the planar robot arm displayed in the top left of Fig. 5 attached to the central black disk and with 4 joints $\theta_{1}, \ldots, \theta_{4}$. We constrain $\theta_{1} \in\left[-\frac{\pi}{2}, \frac{\pi}{2}\right], \theta_{2}, \theta_{3}, \theta_{4} \in[-0.9 \pi, 0.9 \pi]$ and furthermore disallow self-collisions and collisions with the environment (the black rectangle and the floor), yielding $\mathcal{C}_{f} \subset \mathbb{R}^{4}$. The robot now has the task of moving from the start configuration displayed in blue to the red goal joint configuration as shown in the top left figure. We sampled 100000 poses uniformly in $\mathcal{C}_{f}$ using OpenRave [13] and applied our framework. $D C_{\infty}^{2}(X)$ had about 6.2 million triangles and 1.8 million
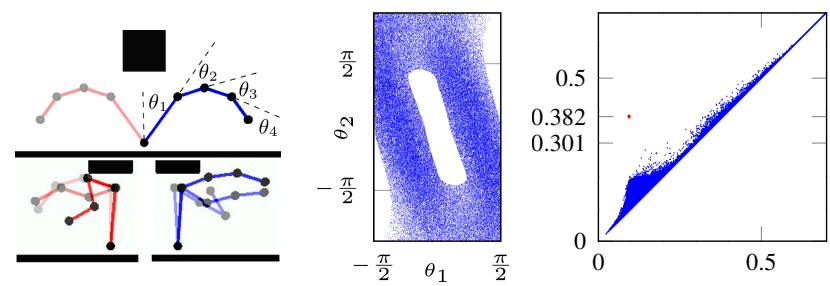

Fig. 5: The top left figure shows the robot arm in start configuration (blue) on the right and in goal configuration (red) on the left. The right figure displays the first persistence diagram for our reconstruction with one red point far above the diagonal. A projection of the samples onto $\theta_{1}, \theta_{2}$ is shown in the middle and an illustration of the difference between the two trajectory classes for $r \in[0.301,0.382]$ is shown in the bottom left figure. In the first trajectory class (in red), the arm is extended to the left when passing under the narrow passage while in the second class (in blue), the arm is extended to the right.

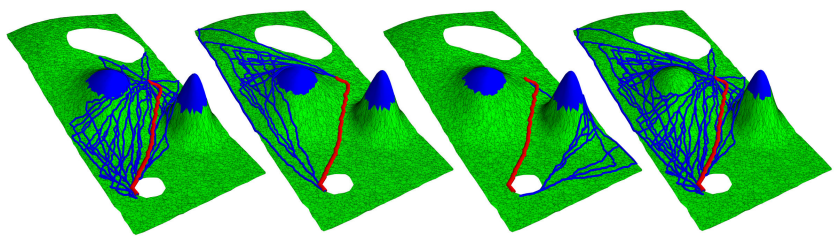

Fig. 6: We display a cost function and classes of trajectories (in blue) depending on a cost threshold and a path $\alpha_{0}$ (in red). For the higher threshold in the rightmost plot, the two classes in the two leftmost figures merge.

edges. The right part of Fig. 5 displays the resulting first persistence diagram which clearly shows that a single homological feature has large persistence in $\mathcal{C}_{f}$. The projection of the joint configurations onto the first two angles, as shown in the middle figure, confirms the existence of a single hole. We computed 1000 edge-paths in $D C_{0.25}$ between the start and end-configuration using 1000 random waypoints as before. For filtration values $r \in[0.301,0.382]$ only two trajectory classes existed. The reduction of the boundary matrix took $0.46 \mathrm{~s}$, while the persistent cycle coordinates for all 1000 paths were calculated in $0.55 \mathrm{~s}$. The Delaunay triangulation in $\mathbb{R}^{4}$ took $251 \mathrm{~s}$, partially due to the increased dimension. Note however that these results are not directly comparable to the $2 \mathrm{D}$ case since methods for 2D Delaunay triangulations in CGAL [1] are especially optimized. We inspected the trajectories in each homology class and found that they were classified according to whether the second link was positioned to the left or to the right of the base link of the arm when $\theta_{1}=0$ as the arm passed the narrow passage (see the bottom left part of Fig. 5). Our framework hence allows the robot to discover the fact that two fundamentally different solution trajectory classes exist.

Filtrations with cost functions: We consider the free configuration space $\mathcal{C}_{f} \subset \mathbb{R}^{2}$ of size 250 by 500 with two obstacles (in white) displayed in Fig. 6. We would now like to distinguish not only between homotopy classes depending on the obstacles in the configuration space, but also discern how trajectories behave with respect to the two peaks of the cost function. The simplicial complex $L_{10, \lambda}(X)$ is displayed for 10000 samples $X$ and height values are determined by the cost function. At cost threshold $\lambda=90$, the top of one of the hills defined by the cost-function is removed from the complex in the rightmost figure (indicated in blue), while at $\lambda=70$ both hills are truncated in the remaining figures. We sampled 100 random paths in this configuration space by fixing the initial 

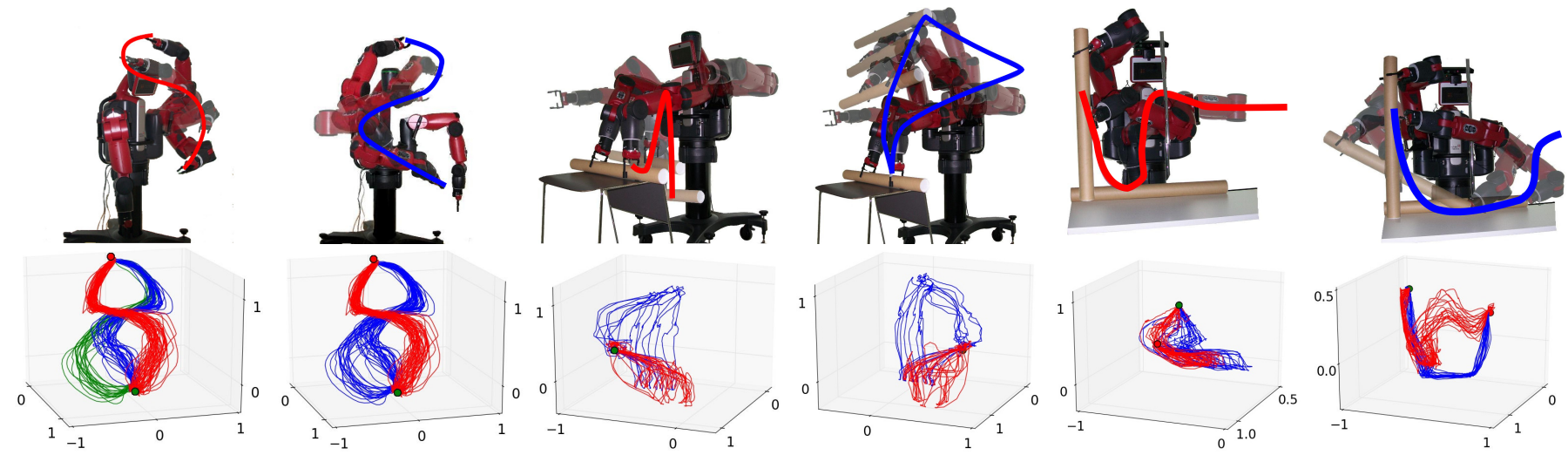

Fig. 7: Baxter robot experiments (E1: column 1-2, E2: column 3-4, E3: column 5-6). Trajectory classes are illustrated in the top row and details are provided in the bottom row. We recorded 97, 18 and 40 trajectories resulting in point clouds with 9594, 3650, 4326 points in dimension 3, 6, and 6, and in filtrations with $0.19,2.72,3.05$ million edges and triangles for E1, E2, E3 respectively. The computation times for (Delaunay triangulation, 2-skeleton and filtration computation, boundary matrix reduction) were $(0.22,0.26,0.12),(41.84,46.95,44.17)$ and $(44.92,51.99,52.44)$ seconds for E1, E2, E3 respectively, while the classification of all trajectories in each experiment took no more than $0.05 \mathrm{~s}$. Bottom row: The first two images show the end-effector trajectories in E1. While we obtain 3 classes for $r=0.08 m$ (first image) the green class merges with the blue one at $r \approx 0.084 m$ (second image, see Fig 8). The $3^{r d}$, $4^{t h}$ and $5^{\text {th }}, 6^{\text {th }}$ image show the right and left hand trajectories for E2 and E3 respectively. The trajectory classes at $r=0.19 \mathrm{~m}$ are indicated in red and blue.

and terminal vertex at the start and end-point of the drawn red reference trajectory and by sampling random waypoints as before. The figure displays example trajectory classes for differing cost filtration values. Note how, at a cost threshold of $\lambda=90$ in the right plot, the two classes depicted in the two leftmost parts of the figure merge.

Baxter robot, 3D and 6D: We now investigate a kinesthetic demonstration scenario where the Baxter robot in Fig. 7 is taught a set of trajectories which we then classify topologically. In the $1^{\text {st }}$ experiment (E1, Fig. 7, column 1-2), the robot is shown two ways to reach from one point above its head to a point in front of its torso. Only one arm is moved in each demonstration while the other arm remains still. We used the end-effector positions of the moving arm to represent trajectories in $\mathbb{R}^{3}$. In the $2^{\text {nd }}$ (column 3-4) and $3^{\text {rd }}$ (column 5-6) experiment E2 and E3, we record the positions of both end-effectors during dual arm manipulations resulting in a $6 \mathrm{D}$ configuration space. In E2, the robot is taught to pick up a cylindrical object with both hands from a table and to move it to one of two positions, one higher and one lower than the table. We also vary the distance between the hands during grasping between demonstrations. The trajectories in E2 are periodic. The motions start with the arms in a rest position on the sides, the object is then grasped and moved, and the rest position is visited again. In experiment E3, the robot moves the same object from a horizontal to a vertical configuration, but a metal bar is located between the robot and the object. Two intuitive motion classes are based on whether the left arm crosses in front of the obstacle, or behind it. Note that, in experiment $\mathrm{E} 1$ and $\mathrm{E} 2$, no obvious obstacles lie directly in $\mathcal{C}_{f}$, but due to the type of demonstrations and the robot's joint limits, the space $X_{r} \simeq D C_{r}(X) \subset \mathcal{C}_{f}$ exhibits interesting voids which we can exploit for classification. We found that trajectories were well-approximated using the described heuristic mapping to nearby edge-paths in $D C_{R}(X)$ (Sec. IV) for $R=0.08 m$ (experiment $\mathrm{E} 1$ ) and $R=0.15 \mathrm{~m}$ (experiment $\mathrm{E} 2$ and E3) respectively. For smaller $r, D C_{r}(X)$ was either not path-connected, or the edge path approximation deteriorated significantly. We hence investigated classifying paths in $X_{r}$ for $r \geqslant R$. Fig. 8 displays how the number of topological
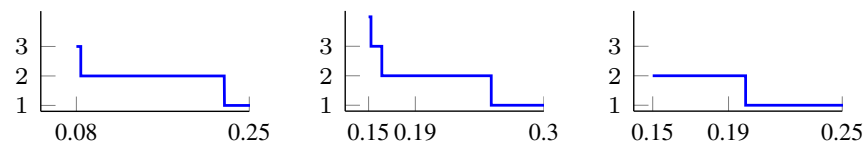

Fig. 8: Number of path classes (vertical axis) vs. filtration value (horizontal axis) for experiments E1, E2, E3 from left to right. All paths exist starting at filtration value $R=0.08 m$ for $\mathrm{E} 1$ and $R=0.15 \mathrm{~m}$ for $\mathrm{E} 2$ and $\mathrm{E} 3$.

trajectory classes changes with varying $r$, and the second row of Fig. 7 displays trajectory classes at various filtration values. In all experiments, there exists a large filtration interval with just two trajectory classes corresponding to the two intuitive classes we just described for each experiment. The first image in the second row of Fig. 7 also illustrates the three classes one obtains in E1 for a choice of $r=0.08 \mathrm{~m}$. More details on the computation times and the sizes of the simplicial complexes is provided in Fig. 7.

\section{CONClusion AND Future Work}

We have explored a novel sampling-based approach to studying homotopy classes of trajectories in general configuration spaces. We believe that our approach could be incorporated with many existing algorithms. For example, the integration of local trajectory optimization based algorithms with our approach which extracts global information about trajectories could be of interest. Another promising future application of our method could be a class-dependent generation of dynamic motion primitives. Several aspects of our approach remain to be investigated more thoroughly, such as the optimal selection of filtration parameters once the persistent cycle coordinates have been computed. While our method scaled well to large sample sets in 2 to 4 dimensions and was applicable also for a smaller set of samples in 6D, Delaunay triangulations and hence $D C_{r}(X)$ have an worst-case complexity of $O\left(n^{\lceil d / 2\rceil}\right)$ [28] in dimension $d$ and sample size $n$. In future work, we hope to investigate also alternative simplicial complexes, such as Vietoris-Rips and (weak) witness complexes [11] which approximate $X_{r}$ and which could be used to mitigate the "curse of dimensionality' in higher dimensions.

\section{ACKNOWLEDGMENTS}

This work was supported by the EU projects TOMSY (ISTFP7-270436) and TOPOSYS (ICT-FP7-318493). 


\section{REFERENCES}

[1] CGAL, Computational Geometry Algorithms Library. http://www.cgal.org.

[2] C. B. Barber, D. P. Dobkin, and H. Huhdanpaa. The quickhull algorithm for convex hulls. ACM Trans. Math. Software, 22(4), 1996.

[3] U. Bauer and H. Edelsbrunner. The morse theory of Čech and Delaunay filtrations. In Proc. of the Thirtieth Annual Symp. on Comp. Geometry, SOCG'14, pages 484:484484:490, New York, NY, USA, 2014. ACM.

[4] U. Bauer, M. Kerber, and J. Reininghaus. PHAT (Persistent Homology Algorithm Toolbox). http://code.google.com/p/phat/.

[5] S. Bhattacharya, V. Kumar, and M. Likhachev. Searchbased path planning with homotopy class constraints. In Proc. of The Twenty-Fourth AAAI Conf. on Artificial Intelligence, Atlanta, Georgia, 11-15 July 2010.

[6] S. Bhattacharya, M. Likhachev, and V. Kumar. Identification and representation of homotopy classes of trajectories for search-based path planning in 3D. In Proc. of Robotics: Science and Systems, 27-30 June 2011.

[7] S. Bhattacharya, D. Lipsky, R. Ghrist, and V. Kumar. Invariants for homology classes with application to optimal search and planning problem in robotics. Electronic pre-print, Aug 2012. arXiv:1208.0573.

[8] A. Billard, S. Calinon, R. Dillmann, and S. Schaal. Robot programming by demonstration. In Springer handbook of robotics, pages 1371-1394. Springer, 2008.

[9] O. Brock and O. Khatib. Real-time re-planning in highdimensional configuration spaces using sets of homotopic paths. In Proc. of the IEEE Int. Conf. on Robotics and Automation (ICRA'O0), 2000., volume 1, pages 550-555. IEEE, 2000.

[10] J. Canny. The complexity of robot motion planning. MIT press, 1988.

[11] G. Carlsson. Topology and data. Bull. Amer. Math. Soc. (N.S.), 46(2):255-308, 2009.

[12] C. Chen and M. Kerber. Persistent homology computation with a twist. In Proc. of the 27th European Workshop on Computational Geometry, 2011.

[13] R. Diankov and J. Kuffner. OpenRAVE: A Planning Architecture for Autonomous Robotics. Technical Report CMU-RI-TR-08-34, Robotics Institute, Pittsburgh, PA, July 2008.

[14] H. Edelsbrunner. The union of balls and its dual shape. Discrete and Comp. Geometry, 13(1):415-440, 1995.

[15] H. Edelsbrunner and J. Harer. Persistent homology-a survey. Contemporary mathematics, 453:257-282, 2008.

[16] H. Edelsbrunner and J. L. Harer. Computational topology: an introduction. AMS Bookstore, 2010.

[17] L. Jaillet and T. Siméon. Path deformation roadmaps: Compact graphs with useful cycles for motion planning. Int. Journal of Robotics Research, 27(11-12):1175-1188, 2008.
[18] L. Jaillet and T. Siméon. Path deformation roadmaps. In S. Akella, N. M. Amato, W. H. Huang, and B. Mishra, editors, Algorithmic Foundation of Robotics VII, volume 47 of Springer Tracts in Advanced Robotics, pages 19-34. Springer, 2008.

[19] S. Karaman and E. Frazzoli. Sampling-based algorithms for optimal motion planning. Int. Journal of Robotics Research, 30(7):846-894, 2011.

[20] L. E. Kavraki, P. Svestka, J.-C. Latombe, and M. H. Overmars. Probabilistic roadmaps for path planning in high-dimensional configuration spaces. IEEE Trans. on Robotics and Automation, 12(4):566-580, 1996.

[21] S. Kim, K. Sreenath, S. Bhattacharya, and V. Kumar. Optimal trajectory generation under homology class constraints. In 51st IEEE Conf. on Decision and Control, 10-13 Dec 2012.

[22] R. A. Knepper, S. S. Srinivasa, and M. T. Mason. Toward a deeper understanding of motion alternatives via an equivalence relation on local paths. Int. Journal of Robotics Research, 31(2):167-186, 2012.

[23] J.-C. Latombe. Robot Motion Planning. Springer, 1991.

[24] S. M. LaValle. Planning algorithms. Cambridge University Press, 2006.

[25] S. M. LaValle and J. J. Kuffner. Rapidly-Exploring Random Trees: Progress and Prospects. In B. R. Donald, K. M. Lynch, and D. Rus, editors, Algorithmic and Computational Robotics: New Directions, pages 293308, Wellesley, MA, 2001. A K Peters.

[26] S. R. Lindemann and S. M. LaValle. Current issues in sampling-based motion planning. In Robotics Research, pages 36-54. Springer, 2005.

[27] E. Masehian and D. Sedighizadeh. Classic and heuristic approaches in robot motion planning - a chronological review. World Academy of Science, Engineering and Technology, 23:101-106, 2007.

[28] P. McMullen. The maximum numbers of faces of a convex polytope. Mathematika, 17(02):179-184, 1970.

[29] B. Morris and M. Trivedi. Learning trajectory patterns by clustering: Experimental studies and comparative evaluation. In IEEE Int. Conf. on Comp. Vision and Pattern Recognition (CVPR'09), pages 312-319. IEEE, 2009.

[30] P. Niyogi, S. Smale, and S. Weinberger. Finding the homology of submanifolds with high confidence from random samples. Discrete and Comp. Geometry, 39(13):419-441, 2008.

[31] J. T. Schwartz and M. Sharir. On the piano movers problem. II. General techniques for computing topological properties of real algebraic manifolds. Advances in Applied Mathematics, 4(3):298-351, 1983.

[32] L. Zhang, Y. J. Kim, and D. Manocha. A hybrid approach for complete motion planning. In Proc. of the IEEE/RSJ Int. Conf. on Intelligent Robots and Systems, (IROS'07), pages 7-14. IEEE, 2007.

[33] Y. Zheng and X. Zhou. Computing with spatial trajectories. Springer, 2011. 